\title{
Is Keratoconus Really Rare?
}

\author{
Nikki Hafezi ${ }^{1,2}$ and Farhad Hafezi ${ }^{1,3}$ \\ 1. ELZA Institute, Dietikon/Zurich, Switzerland; 2. Light for Sight Foundation, Zurich, Switzerland; 3. USC Roski Eye Institute, University of Southern \\ California, LOS Angeles, CA, US
}

DOI: https://doi.org/10.17925/USOR.2017.10.02.91

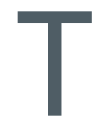

he most used prevalence rate of keratoconus is 1:2,000, which defines the disease as rare. More recent studies suggest that the prevalence is significantly higher than 1:2,000. This increase in prevalence may be explained by the introduction of modern and highly sensitive corneal imaging technologies and a better understanding of geographical variations in the prevalence of keratoconus. The K-MAP study (NCT03115710) will determine the global prevalence of keratoconus in the young population and thus help support the introduction of screening initiatives for this not-so-rare disease.

\section{Keywords}

Keratoconus, cross-linking, prevalence, children, adolescents

Disclosure: Nikki Hafezi and Farhad Hafezi have nothing to declare in relation to this article. This article is a short opinion piece and has not been submitted to external peer reviewers. No funding was received in the publication of this article.

Authorship: All named authors meet the International Committee of Medical Journal Editors (ICMJE) criteria for authorship of this manuscript, take responsibility for the integrity of the work as a whole, and have given final approval to the version to be published.

Open Access: This article is published under the Creative Commons Attribution Noncommercial License, which permits any noncommercial use, distribution, adaptation, and reproduction provided the original author(s) and source are given appropriate credit.

Received: July 21, 2017

Published Online: October 20, 2017

Citation: US Ophthalmic Review, 2017;10(2):91-2

Corresponding Author: Nikki Hafezi, ELZA Institute, Webereistrasse 2,8953 Dietikon, Switzerland. E: nhafezi@elza-institute.com
Despite the successful translation of corneal cross-linking $(\mathrm{CXL})$ into clinical practice for the therapy of progressive ectasia, keratoconus globally still remains a major reason for severe visual impairment in the young population.

Keratoconus is characterized by a biomechanical weakening effect on the corneal stroma. This disease is typically most aggressive in the first three decades of life, and then slows down to an arrest in the fourth to fifth decade. The prevalence of keratoconus has first been investigated in the 1980s, when Kennedy and colleagues detected keratoconus based on irregular retinoscopic reflexes and irregular mires detected with keratometery. ${ }^{1}$ Their study was conducted in the US, and the prevalence reported was 1 in 2,000 patients, which, by definition, is a rare disease. Despite the study being 30 years old, it remains the most cited prevalence rate of keratoconus.

However, a number of more recent publications indicate that the occurrence of this disease might be considerably more frequent than previously estimated. This might be due to the following reasons.

\section{Changes in corneal imaging techniques}

Corneal imaging techniques have evolved since the mid-1980s. In 1985, Klyce and colleagues introduced the concept of modern corneal topography with computer-assisted analysis of the reflection image from Placido photokeratoscope images. ${ }^{2}$ About 20 years later, Scheimpflug imaging technology allowed for a more detailed analysis of both the corneal anterior and posterior surface, coining the term "corneal tomography." ${ }^{3,4}$

\section{Measurement of corneal biomechanics in vivo}

Technology has advanced to help analyze corneal biomechanics and detect early forms of keratoconus with a greater sensitivity. The Ocular Response Analyzer (ORA), originally developed by David Luce in the mid 2000s, was the first of its kind to measure the corneal deformation based on an air puff. ${ }^{5}$ A few years later, high-speed dynamic Scheimpflug imaging allowed for a more detailed analysis of the changes induced by an air puff. ${ }^{6}$ Now, new indices are being developed to allow for a better characterization of keratoconus at an early stage. ${ }^{7}$ More recently, Brillouin microscopy, a means to measure corneal biomechanics in a depth-dependent manner is promising and is in its first clinical stages of development. ${ }^{8}$

\section{Major geographical variations}

The reported prevalence and incidence rates for keratoconus are varied. Geographic, environmental and ethnic factors may be influencing the differing rates; furthermore, interrelations between these factors are also yet to be determined. 
Some of the recently published articles on the prevalence of keratoconus were focused on the Middle Eastern population and have shown a significantly higher prevalence rate than what has been previously reported; their findings show a keratoconus prevalence rate of up to 1:40 subjects. ${ }^{9,10}$

\section{Assessing the global prevalence of keratoconus-the K-MAP study}

The Light for Sight Foundation (Zurich, Switzerland), founded by Nikki and Farhad Hafezi, fosters research initiatives to better understand keratoconus." To draw a more accurate global prevalence rate of keratoconus, Light for Sight has launched the global K-MAP study (NCT03115710). This multi-center study will collect data from children and adolescents in a standardized way and following one common study protocol. We currently have involved 14 sites on five continents. The clinical sites were carefully selected to be able to best represent different geographical regions as well as climate differences. All clinical sites are required to have the same diagnostic equipment in order to be able to accurately compare data. Another consideration for selecting clinical sites is if a patient has a suspicious cornea, the investigator would immediately refer the patient to a corneal specialists for further diagnostics.

The pilot study was conducted in Riyadh, Saudi Arabia, using young subjects and a known geographical region with higher prevalence rates (Middle East); 1,044 eyes of children and adolescents were examined, and the topographical data were assessed by two independent cornea specialists. We found a prevalence rate of $4.79 \%$ (1:21 patients). The manuscript has been submitted for publication.

In conclusion, keratoconus is more common than what is defined by the criteria of a rare disease. The K-MAP study will allow for a more customized geographical approach in the assessment of the need for early screening (and treatment) in the young population to, once and for all, prove that keratoconus should no longer be considered rare. $\square$
1. Kennedy RH, Bourne WM, Dyer JA, A 48-year clinical and epidemiologic study of keratoconus, Am J Ophthalmo 1986;101:267-73.

2. Klyce SD, Computer-assisted corneal topography. High-resolution graphic presentation and analysis of keratoscopy, Invest Ophthalmol Vis Sci, 1984:25:1426-35.

3. Ciolino JB, Khachikian SS, Cortese MJ, Belin MW, Long-term stability of the posterior cornea after laser in situ keratomileusis, Cataract Refract Surg, 2007:33:1366-70.

4. Kim SW, Sun HJ, Chang JH, Kim EK, Anterior segment measurements using Pentacam and Orbscan II 1 to 5 years after refractive surgery J Refract Surg, 2009:25:1091-7.

Luce DA, Determining in vivo biomechanical properties of the cornea with an ocular response analyzer, I Cataract Refract Surg, 2005;31:156-62.

6. Rufer F, Schroder A, Arvani MK, Erb C, [Central and peripheral corneal pachymetry--standard evaluation with the Pentacam systeml, Klin Monbl Augenheilkd, 2005:222(2):117-22.

7. Roberts CJ, Mahmoud AM, Bons JP, et al., Introduction of Two Novel Stiffness Parameters and Interpretation of Air Puff-Induced Biomechanical Deformation Parameters With a Dynamic Scheimpflug Analyzer, J Refract Surg, 2017;33:266-73.
8. Scarcelli G, Yun SH, Confocal Brillouin microscopy for three-dimensional mechanical imaging, Nat Photonics, 2007;2:39-43.

9. Hashemi H, Khabazkhoob M, Yazdani N, et al., The prevalence of keratoconus in a young population in Mashhad, Iran, Ophthalmic Physiol Opt, 2014;34:519-27.

10. Millodot M, Shneor E, Albou S, et al., Prevalence and associated factors of keratoconus in Jerusalem: a cross-sectional study, Ophthalmic Epidemiol, 2011;18:91-7.

11. Light for Sight Foundation, Zurich, Switzerland. Available at: www. lightforsight.org (accessed 3 August 2017). 